\title{
Ferroelectric Liquid Crystal for Use in a New Generation of LCDs
}

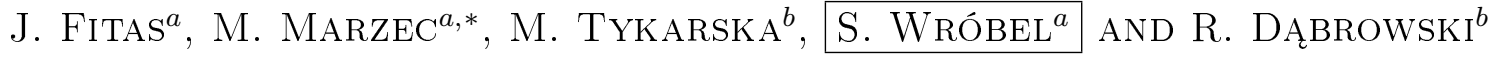 \\ ${ }^{a}$ Institute of Physics, Jagiellonian University, W.S. Reymonta 4, 30-059 Kraków, Poland \\ ${ }^{b}$ Military University of Technology, S. Kaliskiego 2, 00-908 Warszawa, Poland
}

\begin{abstract}
The key to improvements in liquid crystalline displays lies in the continuous synthesis and studies of new kinds of liquid crystalline substances. Among them, ferroelectric compounds are the subject of much attention, due to the potential progresses in switching time, colour depth, and other qualities of liquid crystal displays. In this paper we describe the research of the physical properties of 4-(2-methylbutoxy)phenyl 4-(octyloxy)-benzoate for purposes of its potential application in liquid crystal displays.
\end{abstract}

DOI: 10.12693 /APhysPolA.124.954

PACS: $77.84 . \mathrm{Nh}, 64.70 . \mathrm{M}-$

\section{Introduction}

Liquid crystals (LC, mesomorphic phases, mesophases) are a family of phases of matter between solid crystal and isotropic liquid, well known from their role in the liquid crystal displays (LCDs) [1, 2]. Therefore, they have been the subject of intensive research. Their physical properties such as optical anisotropy (characteristic for crystal) and fluidity (feature of isotropic liquid) are useful in LCD. Switching between transmitting and non-transmitting states of LCD involves switching the orientation of LC molecules within the pixels of the device by external forces, such as an electric field [3-6].

Liquid crystals are characterised by freedom of rotational orientation of molecules and anisotropy of physical properties typical for solid crystals, while sharing the lack of translational freedom of molecules with liquids. LC phases differ from each other by the details of their internal organisation. A single liquid crystalline material exhibits polymorphism showing nematic and smectic phases. When changing temperature LC exhibits a certain sequence of appearance of the particular phases. In the transitions between them, phenomena typical for phase transition are observed, and described in terms of the Landau theory of the phase transition $[4,7]$.

Ferroelectricity of solid crystal means that in absence of external electric field the non-zero polarization exits (spontaneous polarization). In case of LC when molecules are chiral and have non-zero perpendicular component of the dipolar moment, ferroelectricity can arise depending on the alignment of molecules in particular phase, e.g. in the $\mathrm{SmC}^{*}$ or $\mathrm{SmI}^{*}$ phases $[8,9]$. As chiral LC molecules in the tilted phases order themselves into a helical structure, non-zero polarization on trans-layer scale can be observed only when the helix is unwound, e.g. under an electric field or by surface anchoring [10].

${ }^{*}$ corresponding author; e-mail: monika.marzec@uj.edu.pl
The aim of this work was to determine the physical properties of new ferroelectric liquid crystal. Mainly the phase sequence, the tilt angle, spontaneous polarization, and influence of the electric field have been studied $[11,12]$. All these properties are very important from application point of view and should give answer to the question if the compound studied could be used in liquid crystal displays.

\section{Experimental}

The compound studied was 4-(2-methylbutoxy)phenyl 4-(octyloxy)-benzoate (in short 8BOC5). It belongs to the common family of calamitic liquid crystals, due to rod-like shape of its molecule (see Fig. 1). The rigid part is formed of the benzene rings linked by an ester group while the alkyl group forms the flexible part. Under normal conditions the substance is a white powder.

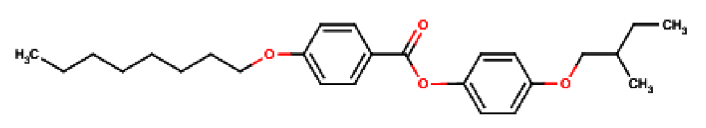

Fig. 1. Molecular structure of 8BOC5.

The research has been conducted using Nikon Eclipse LV100 POL polarizing microscope, PerkinElmer DSC 8000 differential scanning calorimeter, and Novocontrol Microtronic Alpha-A dielectric spectrometer.

The electrooptic ITO cell of $5.1 \mu \mathrm{m}$ thickness has been used in microscopic measurements while to dielectric studies the gold coated cell of $5 \mu \mathrm{m}$ thickness has been applied. The polarizing microscope has been equipped with the heating stage to heat/cool with the certain rate. The digital camera connected to the polarizing microscope has been computer controlled.

Calorimetric measurements have been conducted with different heating/cooling rates to check the stability of the substance studied, find transition temperatures and enthalpy changes at the transitions. Aluminium crucible of $30 \mu \mathrm{l}$ capacity was used. 


\section{Results and discussion}

\subsection{Polarizing microscopy}

The analysis of textures has been performed using ITO surface-aligned electrooptic cell to determine the phase sequence. Microscopic observation of a steadily cooled sample has resulted in the following phase sequence:

$$
\text { Iso } \stackrel{63}{\longrightarrow} \mathrm{N}^{*} \stackrel{62.5}{\longrightarrow} \mathrm{SmA}^{*} \stackrel{42}{\longrightarrow} \mathrm{SmC}^{*} \stackrel{20}{\longrightarrow} \mathrm{Cr} \text {. }
$$

Numbers above arrows are temperatures in the Celsius degrees. As it is seen, the cholesteric phase exists in a very narrow range of temperatures. In fact, during the measurements, no stable cholesteric phase has been achieved, it appears as pretransition region of the $\mathrm{SmA}^{*}$ phase at cooling.

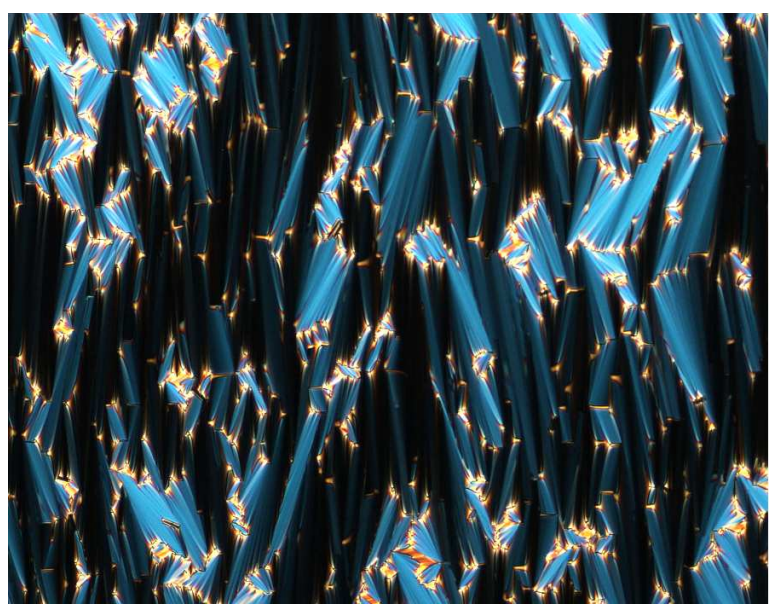

Fig. 2. Texture of the $\mathrm{SmA}^{*}$ phase at $50{ }^{\circ} \mathrm{C}$.

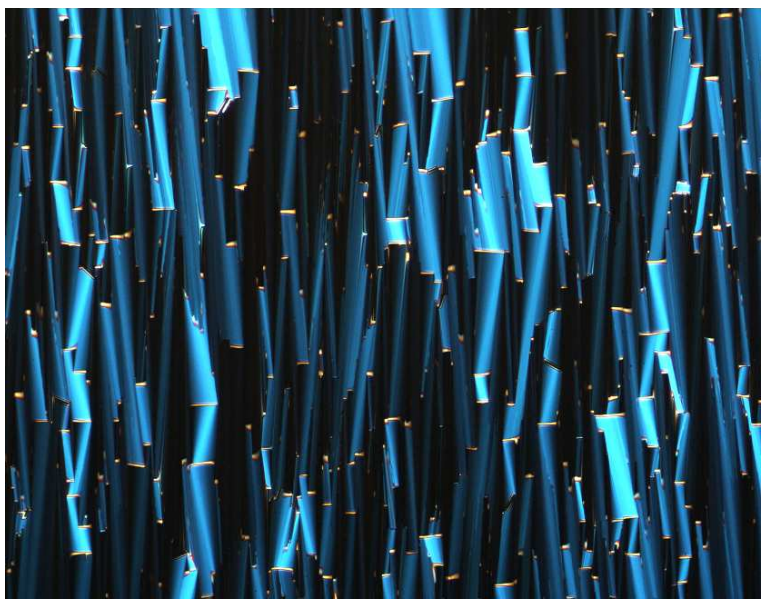

Fig. 3. Texture of the $\mathrm{SmA}^{*}$ phase at $46^{\circ} \mathrm{C}$. The sample has been subjected to field amplitude of $120 \mathrm{~V}$ and frequency of $100 \mathrm{~Hz}$.

Figures 2 and 3 present textures of the $\mathrm{SmA}^{*}$ phase without and with an electric field applied andan influence of an electric field on the texture can be seen, although the $\mathrm{SmA}^{*}$ phase is usually not tilted. Figures $4-6$ show

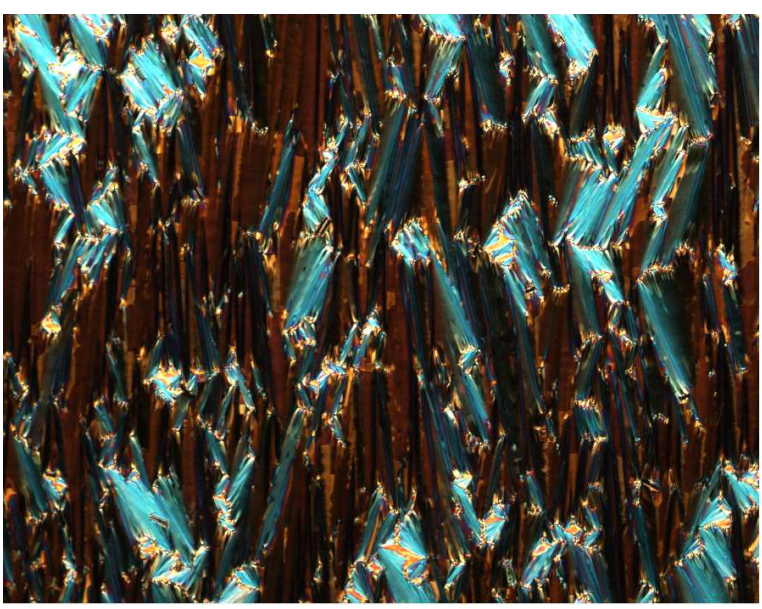

Fig. 4. Texture of the $\mathrm{SmC}^{*}$ phase at $38^{\circ} \mathrm{C}$, without electric field applied.

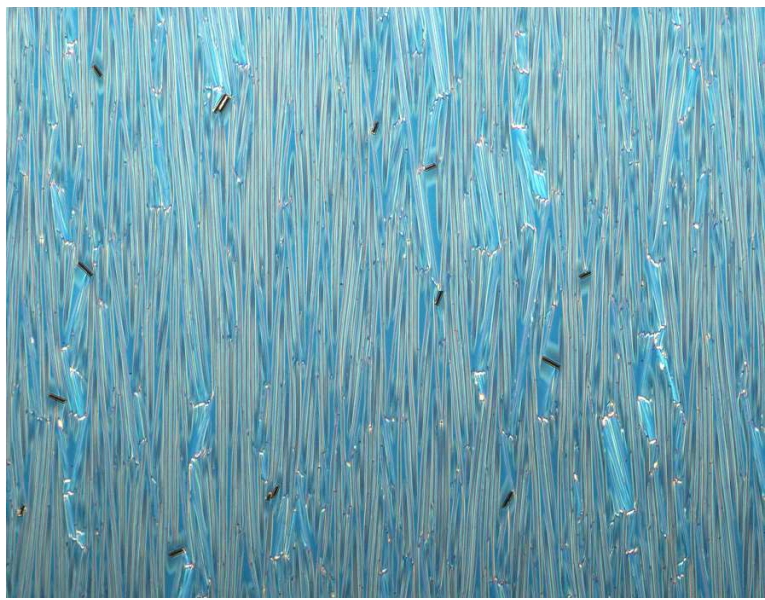

Fig. 5. Texture of the $\mathrm{SmC}^{*}$ phase at $26^{\circ} \mathrm{C}$, under electric field amplitude of $120 \mathrm{~V}$ and frequency of $100 \mathrm{~Hz}$.

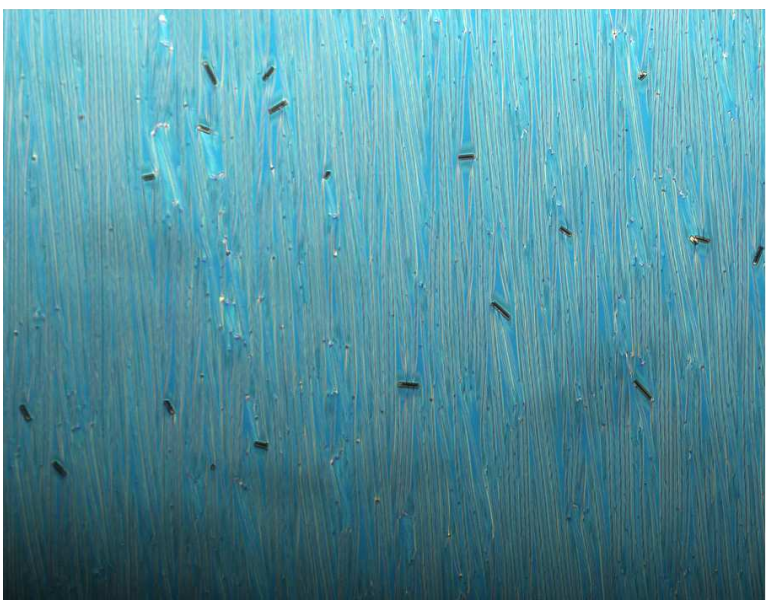

Fig. 6. Texture of the $\mathrm{SmC}^{*}$ phase at $42^{\circ} \mathrm{C}$, after twenty minutes under electric field of $240 \mathrm{~V}$ and $10 \mathrm{~Hz}$. 
textures registered for the $\mathrm{SmC}^{*}$ phase during cooling of the sample. Figure 4 depicts a texture without an electric field applied as the reference to the textures presented in Figs. 5 and 6, where voltage of $120 \mathrm{~V}$ and of $240 \mathrm{~V}$ has been applied, respectively. As it is seen the ferroelectric $\mathrm{SmC}^{*}$ phase orders itself easily under an electric field.

\subsection{Differential scanning calorimetry}

The calorimetric measurements have been performed to determine transition temperatures and enthalpy changes. The critical temperatures of the phase transitions have been calculated from the registered DSC curves using Pyris Software. Based on the DSC results and texture observations the following phase sequence was obtained on heating:

$$
\mathrm{Cr} \stackrel{38}{\longrightarrow} \mathrm{SmC}^{*} \stackrel{43.5}{\longrightarrow} \mathrm{SmA}^{*} \stackrel{58}{\longrightarrow} \mathrm{N}^{*} / \text { Iso. }
$$

As the phases appearing herein are the same as those at cooling, it may be concluded that the substance lacks any monotropic phases.

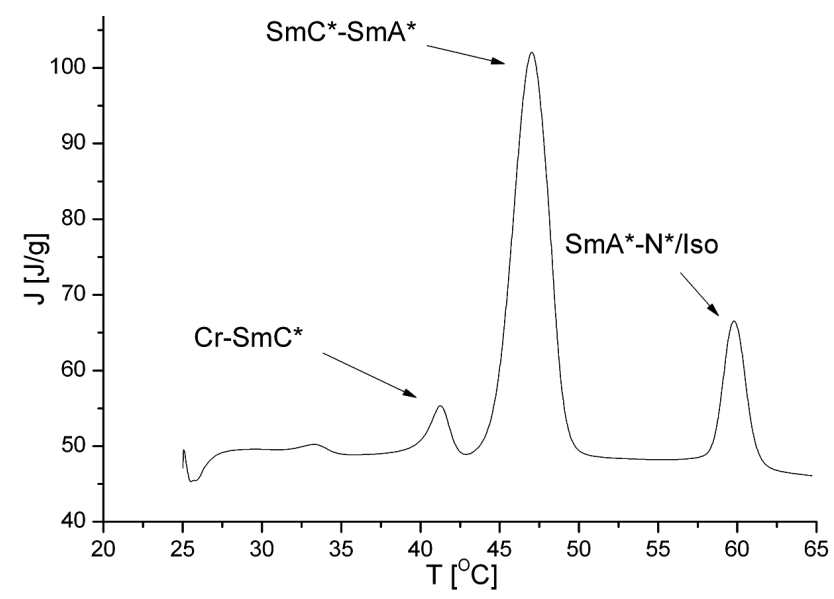

Fig. 7. DSC curve registered at heating with rate equal to $30^{\circ} \mathrm{C} / \mathrm{min}$.

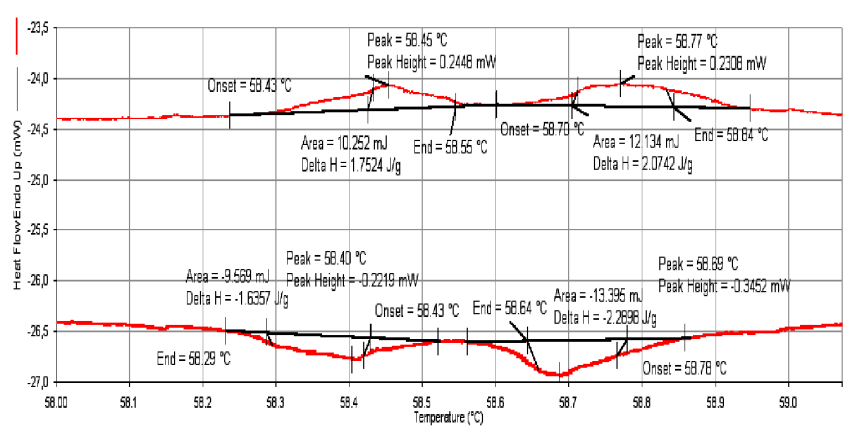

Fig. 8. DSC curves taken at heating (upper curve) and cooling (bottom curve) for enlarged temperature range. Heating and cooling rates are equal to $0.2{ }^{\circ} \mathrm{C} / \mathrm{min}$.

Figure 7 shows DSC curves registered at $30^{\circ} \mathrm{C} / \mathrm{min}$ at heating. Notably, there is a single peak at the transitions
$\mathrm{SmA}^{*}$-cholesteric-isotropic phases. This one peak is split at the heating/cooling rate equal to $0.2^{\circ} \mathrm{C} / \mathrm{min}$. At these conditions the transitions between $\mathrm{SmA}^{*}$ and $\mathrm{N}^{*}$ phase and $\mathrm{N}^{*}$-Iso are well visible, as it is presented in Fig. 8, which confirms the existence of the cholesteric phase in the very narrow temperature range.

\subsection{Spontaneous polarization and tilt angle measurements}

The electric properties of a liquid crystal are crucial to its effectiveness in a display device. The compound studied has been submitted to a series of electric and electrooptic measurements, which began with the measurement of spontaneous polarization. Based on the Landau theory spontaneous polarization (which can be described as the ferroelectric sample's response to an external electric field) behaves in a way predicted by Eq. (1) with critical parameter $\beta$ equal to 0.5 for the second order transition [13]:

$$
P_{\mathrm{s}}=P_{0}\left(T-T_{\mathrm{c}}\right)^{\beta},
$$

where $P_{0}=\left(a / \varphi_{2}\right)^{1 / 2}$ is constant ( $a$ is constant in the Curie-Weiss law and $\varphi_{2}$ is coefficient standing at $P^{4}$ in the series expansion of the Gibbs free energy) and $T_{\mathrm{c}}$ is transition temperature between para- and ferroelectric phases. Spontaneous polarization has been measured versus temperature for different frequencies of triangular wave applied and the results are presented in Fig. 9. As an example temperature dependence of the spontaneous polarization taken at $25 \mathrm{~Hz}$ is shown in Fig. 10 separately. Here the solid line was obtained by fitting Eq. (1) to the results of the measurements. As is seen in Fig. 9, the frequency of the triangular wave applied influence on the temperature dependence of the spontaneous polarization, e.g. it changes its shape, which is also visible in change of the $\beta$ parameter fitted. It turns out that this change is of linear nature, as it is presented in Fig. 11. The straight line here is a result of linear fitting and the $\beta$ parameter extrapolated to zero frequency achieves value of about 0.45 - a value reasonably close to that predicted by the theory for the second order transition. At high frequencies, however, it strays from the model as the relaxation processes fail to keep up with the field changes. Finally, it can be said that observed values of spontaneous polarization are relatively small (several $\mathrm{nC} / \mathrm{cm}^{2}$ ) compared to other liquid crystalline ferroelectric compounds.

The measured values of the tilt angle were in the range of 15-25 degrees. The tilt angle, as related to spontaneous polarization, also behaved in accordance to Eq. (1).

\subsection{Dielectric relaxation studies}

Dielectric properties of the compound have been studied versus temperature. The dielectric spectra have been taken in the frequency range from $1 \mathrm{~Hz}$ to $10 \mathrm{MHz}$. The Cole-Cole model with the conductivity contribution was used to find dielectric parameters [14]:

$$
\varepsilon^{*}=\varepsilon^{\prime}-\mathrm{i} \varepsilon^{\prime \prime}=\varepsilon_{\infty}+\frac{\Delta \varepsilon}{1+\left(\mathrm{i} \omega \tau_{\mathrm{r}}\right)^{1-\alpha}}-\mathrm{i} \frac{\sigma}{\epsilon_{0} \omega},
$$

where $\varepsilon^{\prime}$ is real part (dispersion) and $\varepsilon^{\prime \prime}$ is imaginary 


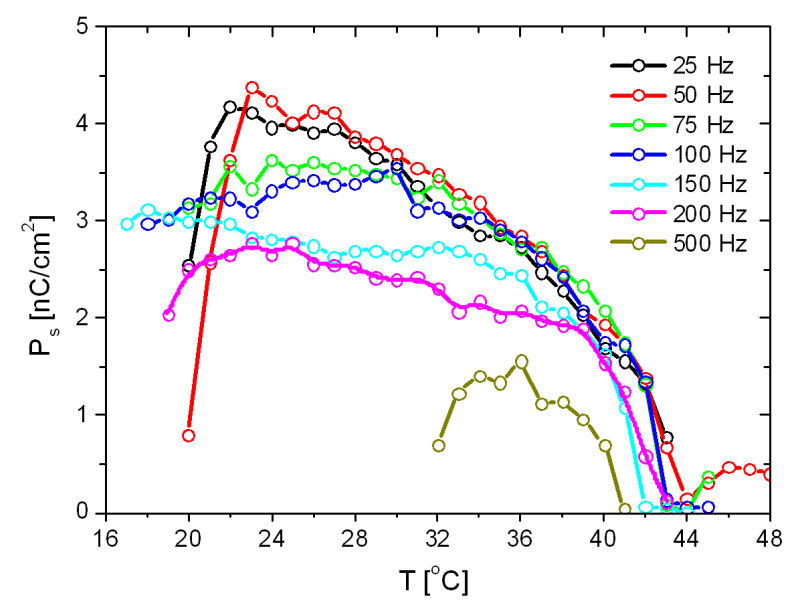

Fig. 9. Temperature dependence of the spontaneous polarization for different frequencies of the triangular wave applied.

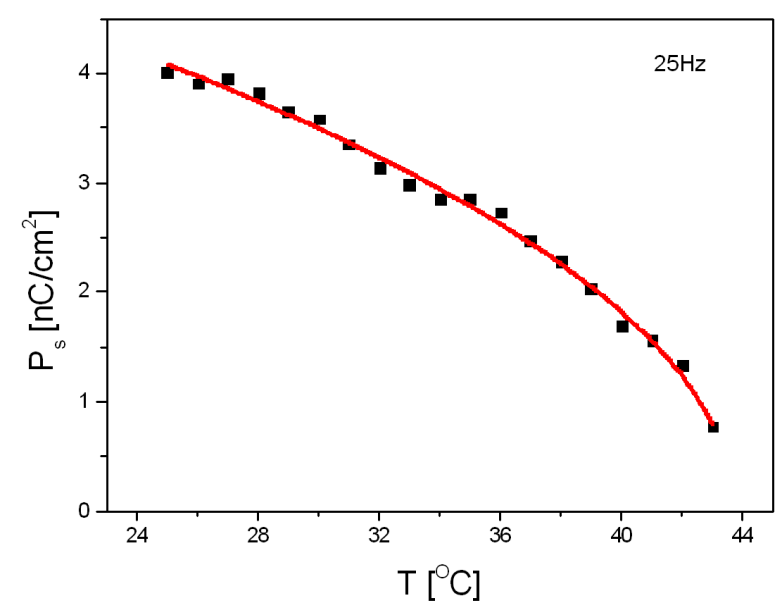

Fig. 10. Temperature dependence of the spontaneous polarization with a theoretical curve fitted, frequency $25 \mathrm{~Hz}$.

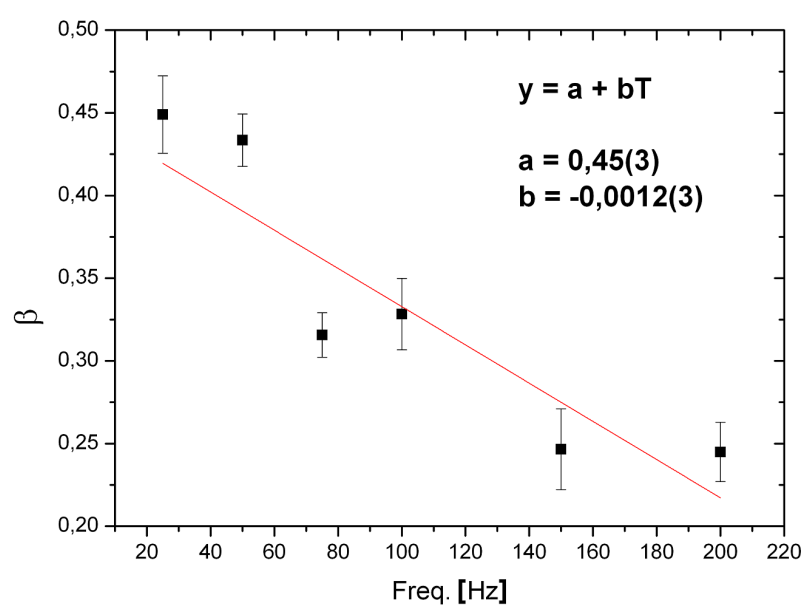

Fig. 11. The exponential parameter $\beta$ versus frequency of electric field applied. part (absorption) of the complex dielectric permittivity $\varepsilon^{*}, \Delta \varepsilon=\varepsilon_{0}-\varepsilon_{\infty}$ is dielectric increment, $\tau_{\mathrm{r}}$ - relaxation time and $\alpha$ is distribution parameter of the relaxation process appearing in the dielectric spectra. $\sigma$ is electric conductivity, $\varepsilon_{0}$ is dielectric permittivity at the low frequency limit while $\varepsilon_{\infty}$ at the high frequency limit of the measurements, $\epsilon_{0}=8.85 \mathrm{pF} / \mathrm{m}$ is dielectric permittivity of free space.

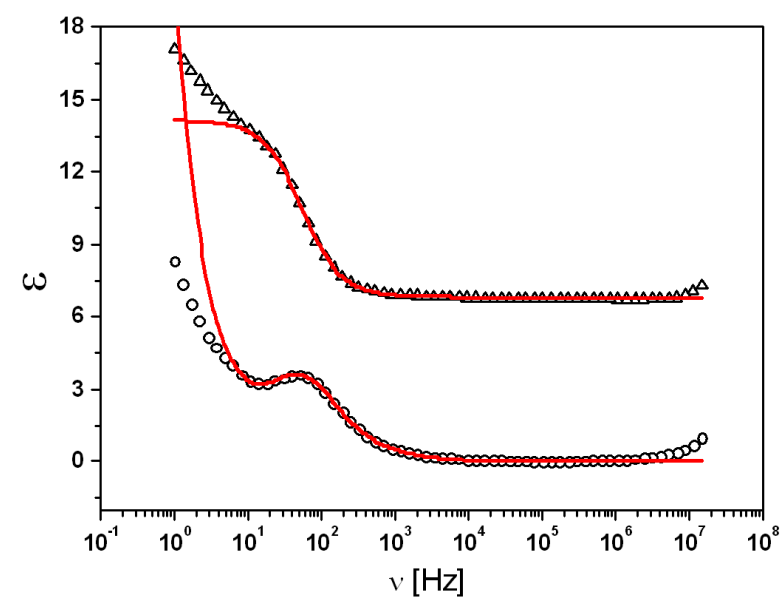

Fig. 12. Dielectric spectrum taken in the $\mathrm{SmC}^{*}$ phase $\left(30^{\circ} \mathrm{C}\right)$, solid lines are fitting results of Eq. (2).

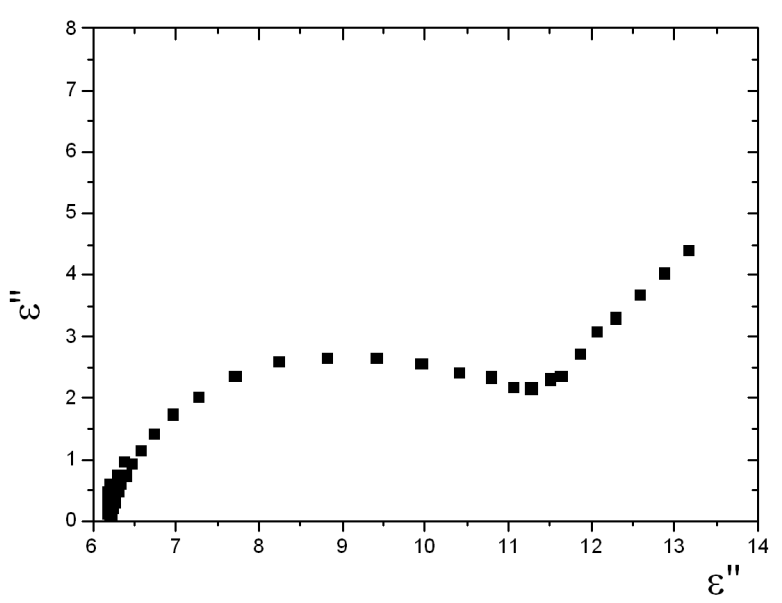

Fig. 13. Cole-Cole diagram plotted for the same data as in Fig. 12.

As an example the dielectric spectra registered in the ferroelectric $\mathrm{SmC}^{*}$ phase for $30^{\circ} \mathrm{C}$ is presented in Fig. 12 while the Cole-Cole plot in Fig. 13. The steep cliff at low frequency is attributed to the influence of an electric conductance. Dielectric increment $\Delta \varepsilon$ and relaxation frequency $\nu_{\mathrm{r}}=1 / \tau_{\mathrm{r}}$ versus temperature are plotted in Figs. 14 and 15, respectively. It is seen that they are almost temperature independent like it is predicted by mean-field model for the Goldstone mode [15]. 


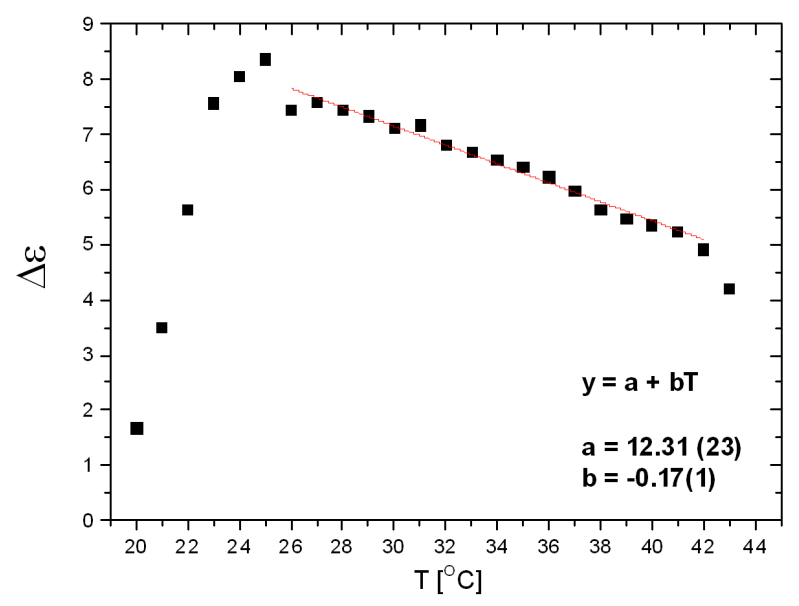

Fig. 14. Temperature dependence of the dielectric increment in the ferroelectric $\mathrm{SmC}^{*}$ phase with linear fit.

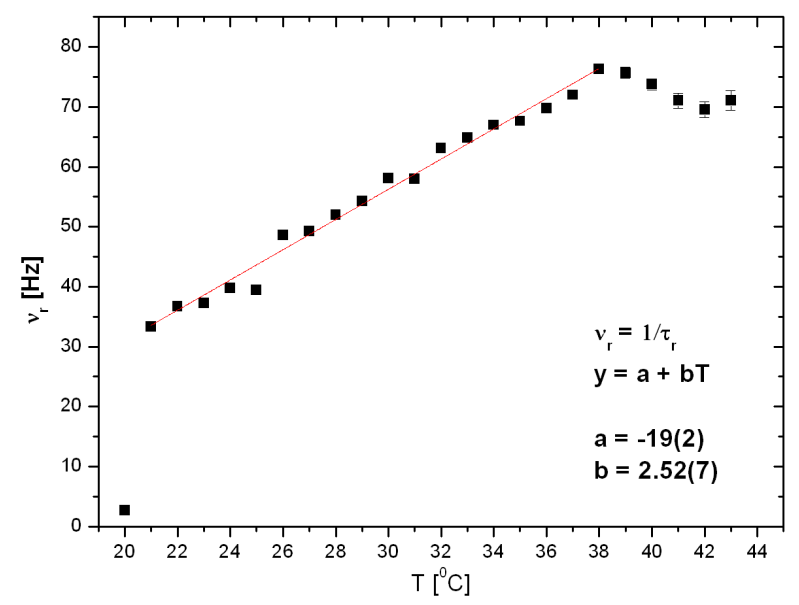

Fig. 15. Temperature dependence of the relaxation frequency of Goldstone mode with linear fit.

\section{Conclusions}

The physical properties of new ferroelectric liquid crystal have been studied. The range of the liquid crystalline phases covered room temperatures but is too narrow to warrant any use for the compound on its own in LCDs. The nematic phase in particular has never been observed in a stable form. The other liquid crystalline phases, however, are quite stable, as long as the sample is surface-aligned.
The spontaneous polarization measured for the compound is relatively small, which means that low amount of energy is needed to switch molecules, which is very important for practical uses. Additionally, it appeared that the frequency of electric fields used must be below $150 \mathrm{~Hz}$. The temperature behaviour of the tilt angle is like for the spontaneous polarization with maximum value $c a .25$ degree.

The dielectric measurements show one relaxation process existing in the ferroelectric $\mathrm{SmC}^{*}$ phase, namely weak Goldstone mode with critical frequency $c a .50 \mathrm{~Hz}$ and dielectric increment $c a$. 7 . Temperature behaviour of this process confirms the mean-field model prediction.

In conclusion, it can be said rather safely that the compound studied is of no use on its own in the LCDs. However, it is promising as one of the ingredients of mixtures.

\section{References}

[1] US Patent, 4462924, Jul. 21 (1984).

[2] US Patent, 3947375, Mar. 30 (1976).

[3] J. Arines, Materials 2, 549 (2009).

[4] I. Dierking, Textures of Liquid Crystals, Wiley-VCH, Weinheim 2003.

[5] A. Adamski, K. Neyts, H. Pauwels, Ferroelectrics 330, 93 (2006).

[6] S.V. Pasechnik, V.G. Chigrinov, D.V. Shmeliova, Liquid Crystals: Viscous and Elastic Properties in Theory and Applications, Wiley-VCH, Weinheim 2009.

[7] V. Minikin, O. Osipov, Y. Zhdanov, Dipole Moments in Organic Chemistry, PWN, Warszawa 1970.

[8] J.K. Ahuja, K.K. Raina, Jpn. J. Appl. Phys. 39, 4076 (2000).

[9] M. Marzec, A. Mikułko, S. Wróbel, W. Haase, in: Dielectric Properties of Liquid Crystals, Eds. Z. Galewski, L. Sobczyk, Transworld, 2007, p. 83.

[10] J. Stoehr, M.G. Samant, J. Electron Spectrosc. Relat. Phenom. 98-99, 189 (1999).

[11] S. Wróbel, S. Hiller, M. Pfeiffer, M. Marzec, W. Haase, Liq. Cryst. 18, 21 (1995).

[12] A. Fąfara, M. Marzec, S. Wróbel, R. Dąbrowski, W. Haase, IEEE Trans. Diel. Electr. Insulat. 8, 3 (2001).

[13] F. Gießelman, in: Self-Organization in Chiral Liquid Crystals, Ed. W. Kuczyński, Scientific Publishers OWN, Poznań 1997, p. 7.

[14] K.S. Cole, R.H. Cole, J. Chem. Phys. 9, 341 (1941).

[15] R. Blinc, B. Žekš, Phys. Rev. A 18, 740 (1978). 